\title{
LES JUIFS DES PROTECTORATS MAROCAINS DANS LES
} ANNÉES 1920:

\section{LA QUESTION DE LA NATURALISATION}

\section{Being a Jew in each Moroccan Protectorate in the 1920's: the nationalization issue}

\author{
Eva Touboul \\ Université Paris Nanterre (Francia) \\ etouboul@parisnanterre.fr
}

Recibido: 27-04-2021 - Aceptado: 09-09-2021

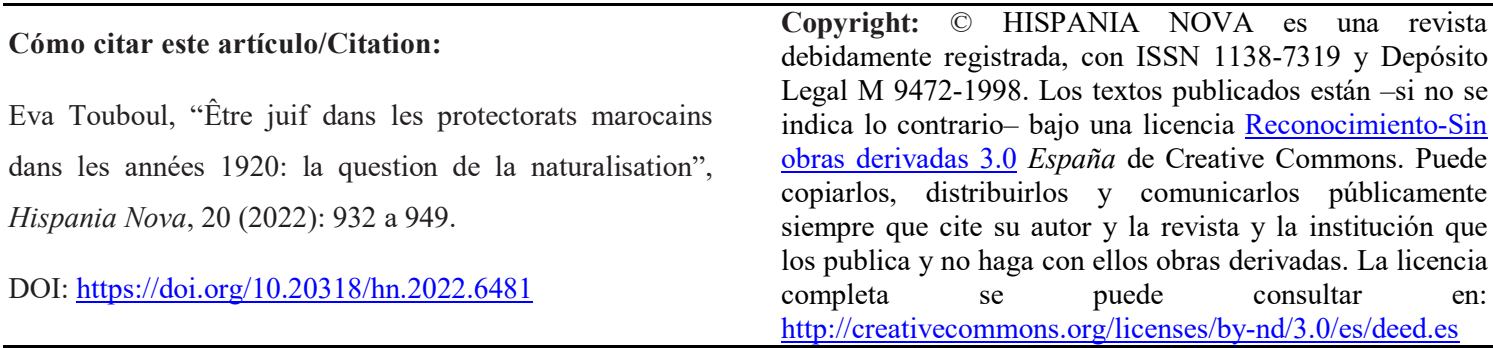

Resumen: En los años 1920 ambos Protectorados francés y español en Marruecos se enfrentan con una misma problemática, la del estatuto administrativo de una población judía globalmente mucho más occidentalizada que los autóctonos musulmanes. Tanto los israelitas franceses, promotores principales de la aculturación de los judíos marroquíes, como los filosefardistas españoles se refieren a la situación de los judíos de Argelia para pedir una nacionalización masiva. Sin embargo, por motivos distintos, las decisiones tomadas por París y Madrid llevarán a mantener a los hebreos de Marruecos en una situación administrativa poco clara.

Palabras clave: Protectorado de Marruecos, Francia, España, Judíos, Sefardismo, Alianza Israelita Universal, Nacionalización

\begin{abstract}
In the 1920's French and Spanish Protectorates in Morocco have to face the same issue: the administrative status of a Jewish population that was much more westernized than the indigenous Muslims. Jewish institutions in France as well as philosephardistic supports in Spain refer to Jewish status in Argelia to call for a massive nationalization. However, for different reasons the decisions made by Paris and Madrid will lead to keeping the Jews from Morocco in an confuse administrative situation.
\end{abstract}

Keywords: Moroccan Protectorate, France, Spain, Jews, Sephardism, Alliance Israélite Universelle, Nationalization 
$\mathrm{Au}$ début du $\mathrm{XX}^{\mathrm{e}}$ siècle, le royaume chérifien se voit écartelé entre différentes influences occidentales, qui prétendent participer au contrôle de ce point d'accès à la Méditerranée et, indirectement, au canal de Suez, et donc au commerce avec l'Asie. Après plusieurs conférences internationales multipartites, s'instaurent deux Protectorats principaux, l'un sous le contrôle de Madrid, l'autre sous celui de Paris, tandis que Tanger est déclarée ville internationale (même si statut exact ne sera précisé qu'après la première Guerre mondiale). Deux administrations différentes (trois, si l'on tient compte du Makhzen qui subsiste tout au long de la période) ont donc en charge l'organisation politique de la vie des habitants de ces territoires. Officiellement, le sultan reste souverain, et les résidents conservent le même statut qu'avant l'instauration des Protectorats. Or trois communautés cohabitent principalement au Maroc : les indigènes ${ }^{1}$ musulmans, les indigènes juifs et les Européens. Si le statut politique des premiers et des derniers est assez clairement défini, celui de la communauté juive est plus flou. Héritage du régime des Capitulations en vigueur dans l'Empire ottoman depuis le XVI ${ }^{\mathrm{e}}$ siècle, un certain nombre de ses membres a le statut de protégé de diverses puissances européennes, qui lui permet d'échapper à la dhimma, l'impôt spécifique lié à un statut juridique d'infériorité par rapport aux musulmans. La Convention de Madrid, en 1880, avait essayé de réguler le droit de protection consulaire, mais avec un effet assez limité.

La population juive du Maroc, composée de descendants de Berbères convertis au judaïsme au début de notre ère, et de descendants de juifs expulsés d'Espagne à la fin $\mathrm{du} \mathrm{XV}^{\mathrm{e}}$ siècle, avait commencé à évoluer sociologiquement dans la seconde moitié du $\mathrm{XIX}^{\mathrm{e}}$ siècle, tout d'abord en s'urbanisant massivement (Michel Abitbol parle de $60 \%$ de la population juive installée dans les principales villes du littoral au début $\mathrm{du} \mathrm{XX}^{\mathrm{e}}$ siècle $^{2}$ ), mais aussi, en recevant, à partir des années 1860, l'influence de l'Alliance Israélite Universelle, héritière de la Haskala, les Lumières juives, qui ouvre à Tétouan

\footnotetext{
${ }^{1}$ Il s'agit du terme habituellement employé par l'administration coloniale pour désigner les habitants autochtones, principalement d'origine berbère ; l'historiographie française actuelle continue d'utiliser ce terme, qui apparaît dans tous les textes juridiques de l'époque. Voir Laure Blevis, «L'invention de "l'indigène", Français non citoyen", dans Histoire de l'Algérie à l'époque coloniale, éd. par Abderrahmane Bouchène et al., 212-218. Paris : La Découverte, 2014.

2 Michel Abitbol, «De la tradition à la modernité: les juifs du Maroc », Judaïsmes : parcours dans la modernité. Diasporas, 27 (2016): 22. doi: https://doi.org/10.4000/diasporas.439
} 
tout d'abord, puis dans les principales villes marocaines, des écoles dont la mission est d'apporter aux communautés israélites du Maghreb la culture et l'éducation françaises, afin de leur permettre d'entrer dans la modernité telle que la concevait l'Occident, tout en maintenant une éducation religieuse traditionnelle à l'attention de leurs élèves, dans un processus d'acculturation.

Lorsque s'instaurent les Protectorats en 1912, les nouvelles administrations se trouvent donc face à une communauté juive disposée à s'intégrer au monde européen, ce qui passe par l'attribution de droits juridiques communs aux ressortissants des deux pays. Dans le prolongement des bouleversements postérieurs à la première guerre mondiale et du bouillonnement de la question des nationalités dans un occident européen et proche-oriental en pleine reconfiguration, c'est dans les années 1920 que les débats autour de la naturalisation des juifs du Maroc apparaissent sur la scène publique, en Espagne comme en France, avec des perspectives et des problématiques différentes.

\section{DES JUIFS DÉSORIENTALISÉS ?}

Comme nous l'avons évoqué plus haut, depuis le début des années 1860, l'Alliance Israélite Universelle (AIU) avait développé dans les principales villes du Maroc un réseau d'écoles dont le programme pédagogique croisait la culture française et l'enseignement religieux traditionnel ${ }^{3}$. Cette incursion du judaïsme "à la française" dans les communautés bien plus traditionnelles du Maroc n'avait pas toujours été accueillie avec bienveillance, dans un premier temps, les rabbins marocains reprochant aux fondateurs de l'AIU de vouloir « déjudaïser » les enfants en leur imposant un système de valeurs exporté d'Europe. C'est ce que rappelle Manuel L. Ortega, zélateur du séphardisme, dans son ouvrage consacré aux juifs du Maroc :

La gran obra de la "Alliance Israélite Universelle" es la que más ha influido en estos últimos tiempos en el progreso intelectual de los hebreos mogrebitas. [...] En 1860 fue fundada en París esta benemérita asociación y dos años más tarde abrió su primera escuela en Tetuán, siguiéndole luego la de Tánger. La de Larache se

\footnotetext{
${ }^{3}$ Michael M. Laskier, The Alliance Israélite Universelle and the Jewish Communities of Morocco $1862-$ 1962, Albany: State University of New York Press, 1983.
} 
estableció en 1902. [...] Los rabinos marroquies se opusieron en un principio al establecimiento de estos centros de enseñanza por recelar de su ortodoxia, excomulgando a los institutores ${ }^{4}$.

Cependant, très rapidement, les fondateurs de l'AIU, relayés par les instituteurs et les rabbins envoyés pour développer les écoles, font la preuve de leurs bonnes intentions : il ne s'agit pas de révolutionner la vie des juifs marocains, mais de leur apporter des outils qui leur permettent de sortir de la vie traditionnelle du mellah, et de former « une génération d'hommes aptes à toutes les fonctions de la société, des citoyens utiles, faisant honneur à la religion qu'ils professent », comme l'indiquait une note d'appel aux contributeurs de $1865^{5}$. Le bilan qui en est tiré dans un article publié par L'Univers israélite (sous-titré « Journal des principes conservateurs du judaïsme ») en 1921 est assez clair : "L'éducation de l'enfant a réagi sur la famille et la société ; les mœurs sont devenues plus policées ; la condition de la femme a été relevée. L'école a transformé les communautés en aiguillant les juifs vers des professions productives et libérales » ${ }^{6}$. Dans un article de 1927 publié dans La Gaceta literaria, José María Millás souligne qu'un équilibre a été trouvé entre modernisation et attachement à la tradition juive, grâce à l'action de quelques grandes figures :

Francia, hace ya algunos lustros que viene realizando una gran labor entre la población hebrea de Marruecos; en casi todas las ciudades importantes hay escuelas de la "Alliance Israélite Universelle". La juventud hebrea recibe en ellas una instrucción solidísima y a la francesa que la hace apta para la vida internacional; quizá había en ello el peligro de la completa asimilación, pero al presente, gracias al movimiento sionista, cuyo portaestandarte es en Marruecos el hebreo español doctor Perl, la juventud hebrea siente un poco la responsabilidad de sus deberes de raza y religión ${ }^{7}$.

L'occidentalisation des juif marocains reste toutefois très relative, si l'on en croit les descriptions faites par les représentants de l'AIU lors de leurs visites des écoles dans les années 1920: ainsi l'inspection menée par de Jacques Bigart, président de

\footnotetext{
${ }^{4}$ Manuel L. Ortega, Los hebreos en Marruecos (facsimil de l'édition de 1919, Málaga: Algazara, 1994): 265.

${ }^{5}$ Cité par B.A., «L'œuvre scolaire de l'Alliance. $1^{\text {ère }}$ partie », L'Univers Israélite, $76 / 24$ (18 février 1921): 559. Voir aussi Abitbol, « De la tradition à la modernité... ».

${ }^{6}$ B.A., “L'œuvre scolaire de l'Alliance. Fin”, L’Univers Israélite, 76/25 (25 février 1921): 583.

7 José María Millás, "Los hebreos del Norte de África”, La Gaceta literaria, 8 (15 avril 1927) : 3. Le docteur Perl dirigeait la revue sioniste Renacimiento de Israel à Tanger.
} 
l'association, en 1926, dont il publie des comptes rendus à la fois dans L'Univers israélite, et dans Paix et Droit, le mensuel de l'Alliance. Il présente la situation des locaux et des moyens d'une dizaine d'écoles de l'AIU, révélant de grandes disparités : face à la modernité de celle de Tanger et l'occidentalisation des élèves de Salé (« on se serait cru plutôt dans un milieu européen qu' au fond d'un obscur mellah » ${ }^{8}$ ), on déplore la misère des installations de Casablanca : «Nos écoles, que j’ai visitées hier, sont mal installées; le nombre des élèves déborde le cadre : ni assez de place, ni assez de lumière, ni assez de confort ». La cause de cette situation est identifiée : ce sont les migrations internes récentes, qui, par ailleurs, ont un impact sur le travail des instituteurs de l'AIU : «Casablanca compte 20.000 juifs dont près des trois-quarts sont venus surtout de l'intérieur, du Sous notamment, et des autres régions du Maroc. L'élément arrivé du sud et de l'intérieur est presque absolument pauvre, et son relèvement sera lent $\gg{ }^{9}$. Mais c'est dans les villes du Protectorat espagnol que la situation est la plus critique :

Je savais par tous les rapports de nos directeurs de Larache que nos écoles de cette ville sont installées dans des conditions déplorables. Mais je ne pouvais me représenter à quel point elles le sont. Je n'entreprends pas la description: salles étroites, sombres, exiguës; je trouve 50 enfants entassés dans une pièce où l'on ne devrait en placer que 20 ou 25. [...] j'ai déclaré aux directeurs et aux membres de la communauté [...] que l'Alliance ne pouvait tolérer un tel état de choses et que nous avions aussi le devoir de fermer des écoles installées dans des conditions aussi antihygiéniques. [À El Ksar] Je m'attendais à trouver des locaux mal installés; on m'avait prévenu, mais la réalité dépasse mon attente : c'est pire encore qu'à Larache, et à pleurer de pitiés pour les enfants qu'on condamne à séjourner, à travailler 6 ou 7 h par jour dans des salles obscures, humides, exiguës ${ }^{10}$.

Alors même que les responsables français de l'AIU s'inquiètent de la situation de leurs écoles et de leurs élèves dans les deux Protectorats, les observateurs espagnols ne cessent de chanter les louanges de son œuvre scolaire, son efficacité et sa

\footnotetext{
${ }^{8}$ Jacques Bigart, « Une mission au Maroc », Paix et Droit, 6/3 (mars 1926) : 5.

${ }^{9}$ Ibid.

${ }^{10}$ Ibid. : 4.
} 
pertinence ${ }^{11}$. Cependant, ils expriment dans le même temps leur inquiétude quant à son effet délétère pour la consolidation de l'influence espagnole dans les territoires placés sous la responsabilité de Madrid : les enfants scolarisés par l'AIU le sont principalement en français. Certes, à l'initiative de l'AIU, et avec le soutien du gouvernement espagnol, depuis 1911 et 1913, respectivement, à Tétouan et Larache, deux enseignants originaires de la Péninsule donnent une heure de cours d'espagnol par jour aux enfants de ces écoles, ce qui, d'après Manuel L. Ortega, reste très insuffisant :

Los esfuerzos patrióticos de estos beneméritos profesores no pueden vencer la influencia francesa. Los hebreos se educan y se instruyen en francés, aprendiendo a admirar y a respetar la gran nación transpirenaica desde sus primeros años. Sin la influencia del hogar donde se habla el castellano, y donde se conserva como algo sagrado el recuerdo de Castilla, pronto desaparecería nuestro idioma de entre los judios marroquíes ${ }^{12}$.

Les Espagnols sont donc inquiets de constater que l'influence française est beaucoup plus puissante que la leur, et relègue, par exemple, la langue espagnole à l'espace privé et familial. Dans les articles de presse consacrés au Protectorat pendant le Directoire de Primo de Rivera, on trouve la demande de la création d'écoles hispanojuives, sur le modèle de celles de l'AIU, qui permettraient d'asseoir la culture espagnole dans des populations que l'on suppose prédisposées à la recevoir, car descendant des juifs expulsés en $1492^{13}$. Manuel L. Ortega va jusqu'à suggérer qu'au-delà d'une imitation, on reprenne carrément les écoles existantes en les hispanisant :

Nosotros entendemos muy bien que siendo universal la Alianza Israelita, y existiendo en otras regiones del mundo colonias de hebreos que no gozan de los beneficios de la instrucción, no resultaría difícil conseguir que España se encargase de las escuelas que posee en el Marruecos español, sosteniéndolas y dotándolas de material de enseñanza. Asi la Alianza podría desarrollar su obra benemérita en otros países, trasladando sus elementos, segura de que las necesidades culturales de los hebreos marroquíes quedaban suficientemente atendidas y garantizadas. Esta debe ser una legítima

\footnotetext{
11 Voir Irene González González, Escuela e ideología en el Protectorado español en el norte de Marruecos (1912-1956). Barcelona: Bellaterra-Casa Árabe, 2015.

12 Ortega, Los hebreos en Marruecos... : 266.

${ }^{13}$ Ce qui est faux, puisque cohabitaient communautés judéo-arabes, judo-berbères et judéo-espagnoles. Voir Haïm Zafrani, Deux mille ans de vie juive au Maroc: histoire et culture, religion et magie. Paris: Maisonneuve \& Larose, 1998.
} 
ambición de España: nadie hallará argumentos suficientes para oponerse a demanda tan justa ${ }^{14}$.

Cette préoccupation des Espagnols pour acculturer les juifs du Maroc s'explique entre autres par le rôle qu'ils leur attribuent dans la modernisation de la zone, et donc l'intérêt pour l'administration espagnole de les attirer vers elle ; ainsi Francisco de Asís Cabrera témoigne en 1923 de la situation de Melilla :

Muchos años he permanecido en la costa norteña de Marruecos, viviendo entre hebreos y nada ha estorbado la armonía entre ellos y cristianos. Melilla da un alto ejemplo a España de fraternidad y de inteligencia entre las dos razas. La libertad, la justicia y la tolerancia mutua, devuelven a los israelitas sus derechos de ciudadanía, arrebatados cínicamente a sus mayores, y ya forman parte, como tales ciudadanos españoles de las Cámaras de Comercio, del Municipio y de otras varias sociedades siendo tan españoles como todos los demás. A los judios, en primer término se debe el grandioso crecimiento urbano de Melilla, en el que han empleado sus capitales. Ellos han sido y son un factor importante en la instrucción española en Marruecos y aún están llamados a desempeñar una mayor acción hispánica si los Gobiernos les atraen en su cariño hacia España ${ }^{15}$.

Comment s'attacher la fidélité des juifs des Protectorats marocains? C'est une question que se posent les philo-sépharades espagnols tout autant que les tenants de l'israélitisme français dans les années 1920, alors même que la présence espagnole est fortement contestée par les rebelles du Rif, objet des préoccupations de la Dictature. Et la réponse qu'ils apportent est la même : la naturalisation.

\section{NATURALISER POUR PLUS D'ÉQUITÉ ? LE MODÈLE ALGÉRIEN EN QUESTION}

En octobre 1870, Adolphe Crémieux, ministre de la Justice du gouvernement de Défense nationale constitué après la défaite de Sedan, promulgue un décret qui accorde la nationalité française aux juifs demeurant en Algérie. Un deuxième décret, le décret Lambrecht, vient corriger un an plus tard ce texte, en limitant sa validité aux seuls juifs

\footnotetext{
${ }^{14}$ Ortega, Los hebreos en Marruecos...: 267

${ }^{15}$ Francisco de Asís Cabrera, "La acción de España: los hebreos", Revista de la Raza, 95 (février-mars 1923) : 23 .
} 
indigènes ${ }^{16}$. Le texte de Crémieux faisait suite à des décennies de débat autour du statut des habitants des trois départements d'Algérie, tout particulièrement ceux désignés comme « indigènes » dans les textes officiels depuis le sénatus-consulte de 1865, à savoir nés sur le territoire algérien avant le début de la colonisation française, ou nés de parents qui résidaient déjà en Algérie lorsqu'elle se produisit. Ces « indigènes » avaient la particularité d'être de nationalité française, mais sans le statut de citoyen et les droits et devoirs qui y sont afférents. Le décret Crémieux, malgré sa rectification postérieure par le décret Lambrecht, a pour conséquence de naturaliser plusieurs dizaines de milliers de juifs d'Algérie, qui se trouvent désormais égaux en droits avec les colons français. Ainsi est parachevée l'œuvre assimilatrice de la France, et les juifs d'Algérie, comme ceux de la métropole, sont désormais vus comme des israélites ${ }^{17}$, à savoir des Français de confession juive.

Cette décision avait été prise sous l'impulsion de l'Alliance Israélite Universelle, dont Crémieux avait été le président, et devait consacrer la désorientalisation complète des populations juives d'Algérie, tout en s'assurant de leur fidélité à la France, pays qui avait fait d'eux des citoyens à part entière. Dans les années 1920, on retrouve donc cette même problématique dans les deux Protectorats du Maroc, dont la population juive semble mûre pour acquérir la citoyenneté d'une des deux puissances européennes. Et en Espagne comme en France, le décret Crémieux reste la référence de l'action à mener en leur faveur. Ainsi Manuel L. Ortega, dans son ouvrage déjà cité, affirme :

La labor que puede y debe realizar España en relación con los hebreos de Marruecos, queda sintetizada en el programa mínimo que a continuación desarrollamos: A) Promulgar una ley parecida a la que dictó en Francia para la Argelia el Ministro M. Crémieux el 24 de Octubre de 1870, concediendo la nacionalidad española, con todos

\footnotetext{
${ }^{16}$ Journal officiel, 9 octobre 1871.

${ }^{17}$ Le terme «israélite» est celui qu'emploie, dans la seconde moitié du XIXe siècle, l'élite socioéconomique juive française pour se désigner. On assiste à un dédoublement de la population juive, entre la communauté naturalisée grâce à l'abbé Grégoire pendant la Révolution française, qui se considère française avant tout, et relègue le religieux au privé, et ceux que l'on désigne comme juifs, fraîchement immigrés en France, et souvent plus attachés aux traditions. C'est cette différence créée par les Français juifs eux-mêmes qui expliquera l'incrédulité de la communauté face à la politique du régime de Vichy pendant l'Occupation : puisqu'ils étaient français, quelle que soit leur confession, il n'était pas imaginable que le gouvernement de leur pays les livre à l'occupant. Dans cet article, nous employons donc « israélite » pour désigner les Français de confession juive, et «juif» pour désigner les membres de la communauté juive.
} 
los derechos civiles y políticos, a los israelitas residentes en el Marruecos español y en nuestras posesiones de África $[\ldots]^{18}$.

Pour les tenants de cette naturalisation massive, il s'agit là d'une évidence, de l'aboutissement d'une évolution. Ainsi Yomtob Semach, représentant de l'AIU au Maroc, signale en 1927 qu'après 15 ans de présence française dans le Protectorat, plus rien ne différencie les juifs des Européens, «rien ne les sépare plus, rien ne les divise plus... que la législation, qui met une barrière morale entre eux ; elle considère le juif comme un indigène replié sur lui-même, drapé dans sa dignité, lent à s'épanouir, à s'adapter $\gg{ }^{19}$. Plus encore, les juifs du Maroc souhaitent s'intégrer pleinement à la société française : «le juif ne tient nullement à son statut personnel, ses principes religieux peuvent s'accommoder des lois civiles communes à tous, il ne demande qu'à se confondre avec la masse des citoyens ${ }^{20}$.

Pour les publicistes espagnols, derrière la revendication de l'octroi de la nationalité à tous les juifs du protectorat du Maroc se cache une intention double : il s'agit d'attirer vers l'Espagne un secteur de la population locale qui occupe une place importante dans la structure économique locale, et surtout de le faire plus vite que la France, pour qui, lassés d'attendre que l'Espagne réponde à leurs attentes, pourraient se tourner vers une France à laquelle les années de scolarité dans les établissements de l'AIU les attachent de façon évidente :

Tenemos muchos amigos de Tetuán, Larache y Alcazarquivir que siguen ansiando la nacionalidad española que no les otorgan. ¿Por qué? ¿Qué mal hay en ello? A menudo nos hablan de este bello deseo, que es flor preciada y exquisita de su corazón. Plumas tan autorizadas en este problema como son las de D. Ignacio Bauer, "Colombine", Cansinos Assens [...] deben volver a templar sus péñolas y volver a pedir a nuestros Gobiernos, tolerantes y comprensivos, la tan ansiada nacionalidad española para los sefardies, para esos hombres que llevados de un auténtico amor a España, anhelan ponerse bajo los pliegues de nuestra bandera. [...] Y mientras Francia es una realidad palpable en esta atracción del espíritu sefardi, que es espíritu de España, nosotros seguimos sin

\footnotetext{
${ }^{18}$ Ortega, Los Hebreos en Marruecos ... : 343-344.

${ }^{19}$ Yomtob D. Semach, « L'avenir des Israélites Marocains », Paix et Droit, 7/6 (juin 1927) : 10.

${ }^{20}$ Ibid.
} 
abordar de lleno, cual es nuestro deber, esta santa y noble causa de redención de nuestros propios hermanos. Conviene llevar a cabo esta política para servir así altos destinos y cumplir nuestra misión en el Mogreb español. Démosles, en hora buena, carta de nacionalidad sin esas trabas burocráticas que les ponen, y ella será como una limpia ejecutoria de abolengo y de realeza para su fin espiritual, material y moral $^{21}$.

Dans cette Espagne placée sous le régime dictatorial conservateur de Primo de Rivera, on trouve aussi une sorte de nécessité de racheter, par cette naturalisation, le «péché originel » de la décision d'expulsion des juifs prise par les Rois Catholiques en 1492. C'est une référence que l'on retrouve dans de très nombreux articles de la presse philosépharade espagnole tout au long des années 1920, le devoir pour l'Espagne de compenser le tort fait aux ancêtres des sépharades d'aujourd'hui en les incluant à nouveau dans la communauté nationale, et en en faisant des citoyens espagnols de plein droit. Ce qui est particulièrement intéressant dans les arguments avancés par les intellectuels péninsulaires impliqués dans cette défense de la naturalisation, c'est qu'ils sont centrés sur l'intérêt, tant économique que moral et symbolique, qu'y trouverait $1^{\prime}$ Espagne ${ }^{22}$. Or les journalistes et responsables de l'AIU qui publient dans la presse communautaire française ont un point de vue plus pragmatique et juridique, en lien avec le statut administratif des juifs résidant dans le Protectorat et à Tanger, ville sous statut international.

La situation légale des juifs du Maroc avait en effet évolué depuis la mise en place des Protectorats. Ils n'avaient jamais été considérés comme des sujets marocains de plein droit pour des questions religieuses; la mise en place des administrations espagnole et française les avait de fait placés sous la protection de ces deux nations, mais ils restaient, suivant la terminologie en vogue à cette époque, heimatlos, apatrides, et relevaient d'une juridiction religieuse spécifique. Yomtob Semach tout comme Alfred Berl, directeur de Paix et Droit, s'étonnent dans plusieurs numéros de ce mensuel de l'inaction de la France et appellent à une réaction rapide, qui permette de protéger les cent mille juifs marocains :

\footnotetext{
${ }^{21}$ Felipe Verdejo Iglesias, "Los sefardíes añoran España en su destierro - Conducta que debe seguir España en Marruecos y Oriente", Revista de la Raza, 163 (avril 1929) : 26.

22 cf. Eva Touboul Tardieu, Séphardisme et hispanité : l'Espagne à la recherche de son passé (19201936), Paris : PUPS, 2009.
} 
Quel homme d'État digne de ce nom ne s'inquièterait, au sein d'un pays de protectorat, qui n'est pas sans susciter l'envie des nations rivales, de laisser flotter une population sans droits, mais aussi sans devoirs, au gré des intérêts changeants, des conflits internationaux? N'y a-t-il pas là un danger, sur lequel il serait imprudent de fermer les yeux? [...] Pourquoi ne pas ouvrir l'accès à la Cité à la fraction du judaïsme marocain la plus digne de l'obtenir ${ }^{23}$ ?

Autre argument en faveur de la naturalisation des juifs du Maroc : la question des équilibres nationaux dans les protectorats du Maroc et de Tunisie. Dès le début des années 1920, on observe une certaine préoccupation des autorités françaises par rapport à la situation démographique dans ces deux pays. Outre la population dite " indigène », musulmane et juive, on y trouve des ressortissants français mais aussi d'autres nations. Or ces derniers se trouvent être plus nombreux que les Français en Tunisie, tandis qu'au Maroc, le rapport de force est plus équilibré. C'est ce qui amène le Parlement à prendre plusieurs mesures de naturalisation des ressortissants étrangers de Tunisie tout au long des années 1920 (tout particulièrement la loi de décembre 1923). Or rien de tel pour le Maroc ; c'est ce qui pousse, entre autres, un député de Constantine à réclamer une décision rapide du gouvernement français, dans un contexte de dénatalité qui ne laisse pas espérer que la métropole puisse rapidement « exporter » des nationaux ${ }^{24}$.

Cependant, si ces revendications se répètent tout au long des années 1920, rien ne semble être fait, ni par l'Espagne, ni par la France, malgré les arguments développés par les partisans de la naturalisation massive des juifs du Maroc. Comment expliquer cet attentisme apparent?

\section{DES OBSTACLES ET UNE TIMIDE TENTATIVE}

On l'a compris, les autorités espagnoles et françaises sont globalement réticentes à l'idée d'imiter l'expérience du Décret Crémieux, pour des raisons différentes. Dans le cas de Paris, tout d'abord, la naturalisation collective des Juifs d'Algérie n'a pas laissé une bonne impression. Elle a tout d'abord été rejetée par les secteurs les plus

\footnotetext{
${ }^{23}$ Alfred Berl, « Au Maroc », Paix et Droit, 8/4 (avril 1928) : 2.

${ }^{24}$ «Les naturalisations au Maroc et en Tunisie », L’Univers Israélite, 81/14 (25 décembre 1925) : 374 ; Berl, « Au Maroc »... : 2.
} 
traditionnalistes de la communauté juive autochtone, qui redoutaient une déjudaïsation de leurs coreligionnaires naturalisés, et donc placés sous influence française. Par ailleurs, elle a suscité une certaine colère des indigènes musulmans, car elle créait un déséquilibre statutaire au sein de la population ${ }^{25}$. Par ailleurs, on considère que les flambées d'antisémitisme que connurent, dès la fin du XIX ${ }^{\mathrm{e}}$ siècle, les départements d'Alger et d'Oran sont à relier à l'application très large du décret Crémieux. Face à ces objections, on lit sous la plume de Semach et Berl des suggestions contradictoires : Semach, qui vit au Maroc, revendique une naturalisation de masse, qui assoie la présence française au Maroc et son influence culturelle et économique ; il craint par ailleurs que la complexité des démarches à entreprendre pour une naturalisation individuelle rebute les intéressés ${ }^{26}$. Berl lui préfère une naturalisation sélective :

Il ne s'agit pas de décréter la nationalisation en bloc; il suffirait de tailler généreusement dans l'élément israélite des tranches où l'on pourrait recruter des nouveaux mais bons et loyaux citoyens. Que l'on accueille l'élite intellectuelle, rien de mieux, mais sans exclusivisme à l'égard des autres classes sociales. [...] De bons employés, des ouvriers qualifiés, possédant la langue française et pouvant démontrer qu'ils ont vécu dans une ambiance française [...] ne seraient pas de moins bonnes recrues pour la France que certains diplômés des différentes écoles. Leur nombre, en outre, serait bien supérieur ; un tel appoint serait vraiment précieux ${ }^{27}$.

Berl parle cependant d'une naturalisation par catégories, ce qui sous-entend que lui non plus ne souhaite pas que les démarches soient à entreprendre de manière individuelle.

Autre argument contre ce processus, d'après Berl: les autorités françaises craindraient de créer un déséquilibre avec le Protectorat tunisien, plus ancien (1881). À cela, il répond en deux points : outre la possibilité d'appliquer le plus favorable des deux textes aux deux protectorats, «si les juifs tunisiens sont depuis plus longtemps des protégés français, ceux du Maroc ont bien plus rapidement évolué, et, en fait, ils sont

\footnotetext{
${ }^{25}$ Voir Laure Blevis, «La citoyenneté française au miroir de la colonisation : étude des demandes des "sujets français" en Algérie coloniale ", Genèses, 53 (2003/4) : 25-47. https://www.cairn.info/revuegeneses-2003-4-page-25.htm, et Florence Renucci, «Le débat sur le statut politique des israélites en Algérie et ses acteurs (1870-1943) ", Contributions du séminaire sur les administrations coloniales (2009-2010), 31-49. Paris, 2010. https://halshs.archives-ouvertes.fr/halshs-00599296

${ }^{26}$ Yomtob Semach, «L'avenir des israélites marocains. II », Paix et Droit, 8/6 (juin 1928) : 4-6.

${ }^{27}$ Berl, « Au Maroc »... : 2.
} 
peut-être plus mûrs pour l'exercice des droits et l'accomplissement des devoirs civiques $»^{28}$. Enfin Berl exprime sa crainte de voir ses coreligionnaires, déçus par l'impossibilité d'appartenir à la nation française, se tourner vers ce qu'il considère comme «les solutions du désespoir ", à savoir le communisme et le sionisme. Dans un article paru dans Chalom, la revue de l'Union Universelle de la Jeunesse Juive, Edmond Fleg renchérit :

Les Juifs marocains ne songent pas plus à revendiquer les droits d'une minorité nationale qu'à aller s'établir en Palestine. Mais les éduquer en Français pour leur refuser tout accès à la communauté française, serait faire d'eux, à brève échéance, un groupe déclassé, dont certains éléments ne tarderaient pas à chercher ailleurs le statut d'une vie nouvelle ${ }^{29}$.

On sait que le sionisme avait en effet quelque écho dans le Protectorat, au point que le Maréchal Lyautey, Résident Général de 1912 à 1925, s'en était lui aussi inquiété et s'était associé aux représentants de l'Alliance pour lutter contre ce qui était considéré comme de la propagande étrangère, mais cela n'infléchit pas son opinion sur la situation administrative des juifs du Maroc ${ }^{30}$.

Lyautey était en effet loin d'être favorable à une naturalisation de masse des juifs du Protectorat. Ainsi qu'il l'avait exprimé aux directeurs de différentes écoles de l'AIU dès sa prise de fonction, et qu'il mit en application, il pouvait accepter de naturaliser des élites parfaitement occidentalisées, mais se refusait à accorder le même droit à "la tourbe" ${ }^{31}$. On ne peut écarter, chez les autorités coloniales, un fond d'antisémitisme qui ne leur permet pas d'accepter l'idée d'une occidentalisation rapide de ces populations, ni celle que permettrait la naturalisation. Ainsi dans une conférence de 1920 souvent citée, le Directeur de l'Enseignement au Maroc indiquait que s'il fallait se réjouir de l'évolution rapide des juifs du Protectorat, il fallait cependant la contenir, au risque que « de l'humble chrysalide noire [sorte] un papillon que grise la brusque lumière et qui,

\footnotetext{
28 Ibid.

${ }^{29}$ Edmond Fleg, « Le Judaïsme au Maroc », Chalom, 39 (mai-juin 1928) : 5.

${ }^{30}$ Mohammed Kenbib, « La politique indigène du général Lyautey et les juifs marocains (1912-1925) », in De l'Atlas à l'Orient musulman, éd. par Dominique Avon et al. (Paris: Karthala, 2011) : 79

${ }^{31}$ Ibid. : 69.
} 
mal préparé à son bonheur, deviendrait vite un papillon nouveau riche et jeune israélite, en somme un très vilain et très gênant insecte $»^{32}$.

Paradoxalement peut-être, le Directoire militaire du général Primo de Rivera prit une décision assez inattendue en promulguant, en décembre 1924, un Décret qui permettait aux Sépharades de demander la nationalité espagnole ${ }^{33}$. Le texte n'était pas aussi explicite, puisqu'il faisait référence à

antiguos protegidos españoles, o descendiente de éstos, y en general, individuos pertenecientes a familias de origen español que en alguna ocasión han sido inscritas en Registros españoles, y estos elementos hispanos, con sentimientos arraigados de amor a España, por desconocimiento de la ley, y por otras razones ajenas a su voluntad de ser españoles, no han logrado obtener nuestra nacionalidad. Muchos de ellos restan con la errónea creencia de que la poseen y de que para su disfrute sólo les falta algún requisito externo que con equivocado empeño solicitan. Otros esperan una naturalización en masa de la colectividad de hispanófilos militantes a la que pertenecen, y son muchas las cosas en que esta misma condición de aspirantes a la nacionalidad española les hace encontrarse con ninguna ${ }^{34}$.

L'allusion est parfaitement transparente aux anciens protégés de l'Espagne, qui n'avaient pas tous connaissance du fait que la mise en place du Protectorat s'était faite au détriment de leur ancien statut. Ce texte prétend donc offrir un cadre à leur régularisation juridique, dans une période limitée au 31 décembre 1930. Notons cependant que ce texte ne s'adresse pas exclusivement aux sépharades du Maroc, mais aussi - et surtout - à ceux établis dans l'ancien Empire ottoman et que les différents traités postérieurs à la première guerre mondiale ont rendus apatrides, du fait des critères de nationalité établis par chacun des pays nés du démantèlement de l'Empire. Par ailleurs, indirectement, ce texte risquait de créer une différence de statut entre les différentes communautés juives présentes au Maroc, puisqu'il n'ouvrait la possibilité d'acquérir la nationalité espagnole qu'aux individus pouvant accréditer leur origine

\footnotetext{
${ }^{32}$ Cité dans ibid. : 73.

33 cf. Davide Aliberti, Sefarad: una comunidad imaginada (1924-2015), Madrid: Marcial Pons, 2018.

34 «Real decreto concediendo un plazo que, improrrogablemente, finará en 31 de Diciembre de 1930, para facilitar la naturalización de individuos de origen español que vienen siendo protegidos como si fueran españoles, y aclarando para ello el sentido de los preceptos legales que expresamente se mencionan », BOE-A-1924-11729.
} 
espagnole, ce qui laissait donc de côté les communautés judéo-arabes et judéo-berbères. Enfin, contrairement au décret Crémieux qui restait la référence, il ne s'agissait en aucun cas d'une naturalisation de masse, mais bien de la facilitation des démarches individuelles devant aboutir à la naturalisation : ainsi, le texte envisageait de dispenser les candidats d'un déplacement dans la Péninsule pour finaliser leurs démarches, et, surtout, d'une obligation de résidence en Espagne. On peut s'étonner de cette exception, mais elle est expliquée, entre les lignes, dans le préambule du texte : «esos elementos [...] han de resultar propicios mediante la naturalización a difundir [nuestro idioma] en beneficio de nuestras relaciones culturales en países lejanos en los cuales forman colonias que pueden ser de verdadera utilidad para España ${ }^{35}$.Il s'agit bel et bien d'utiliser ces Espagnols « du monde entier » en faveur de la consolidation d'un empire culturel qui ferait de l'Espagne une puissance mondiale susceptible de rivaliser avec la France ou la Grande-Bretagne, mais dont l'influence reposerait non sur des possessions territoriales, mais sur des communautés de nationaux nés à l'étranger, et que la naturalisation finirait de fidéliser.

Fait étonnant, même après 1924, dans la presse espagnole comme dans la presse communautaire juive francophone, on continue de trouver des articles demandant la naturalisation des juifs du Maroc ; ainsi Saül Mezan, qui en février 1928, proclame que “dans la zone espagnole du Maroc il est nécessaire que tout juif parlant l'espagnol puisse acquérir (ou plutôt recouvrer) sans difficulté aucune la nationalité espagnole ${ }^{36, "}$ ou Rodolfo Gil Benumeya écrivant en janvier 1931: «Junto a la acción pasada, la acción futura. Quedan muchos, infinitos problemas. Son los más urgentes: naturalización de los hebreos de Melilla, Ceuta y zona española que lo deseen, sin ninguna formalidad burocrática, automáticamente $»^{37}$. Il semble donc que l'on n'ait donné que peu de publicité à ce décret : certaines légations ont continué de distribuer des passeports espagnols à ceux qui n'avaient que le statut de protégés, tandis que l'on n'a pas cherché à expliquer aux communautés judéo-espagnoles du Maroc l'intérêt

\footnotetext{
35 Ibid.

36 Saül Mezan, «L'Espagne et les Juifs de langue espagnole », L’Univers israélite, 83/22 (17 février 1928) : 679 .

${ }^{37}$ Rodolfo Gil, « El año sefardí. 1930 », La Gaceta Literaria, 97 (1 ${ }^{\mathrm{er}}$ janvier 1931): 20.
} 
qu'avaient leurs membres à se prévaloir de ce texte. C'est le bilan qu'en tire le Dr. Perl en 1931 :

Hay que reconocer que es muy reducido el número de individuos que se han aprovechado de las facilidades que este Decreto les brindaba ; pero tal pasividad es debida, más que nada, a la falta de publicidad y difusión que la soberana disposición ha tenido y a la circunstancia de que muchas personas no estiman necesaria la naturalización, ya que los naturales de nuestra Zona gozan dentro de ella y en el extranjero de los mismos derechos que los españoles peninsulares $^{38}$.

Pourquoi le Directoire de Primo de Rivera a-t-il fait le choix de ne pas plus et mieux diffuser ce texte, tant au sein des populations concernées qu'auprès des représentations diplomatiques espagnoles? Il est probable que Madrid ait craint un engouement auquel elle n'était pas en mesure de faire face. En effet, si le texte dispensait les candidats à la naturalisation d'un voyage dans la Péninsule pour réaliser les démarches, et d'une installation sur le territoire national, il ne les en empêchait pas. De plus, les obligations militaires auxquelles devaient se soumettre les jeunes naturalisés pouvaient apparaître comme une incitation à l'immigration, ainsi que le signalait un rapport remis au ministère de l'Intérieur en $1930^{39}$. Or, comme dans l'administration française, un fond d'antisémitisme ne peut être écarté, en ce sens où l'antijudaïsme chrétien traditionnel était encore fortement ancré dans la population espagnole, et faisait craindre à des autorités elles-mêmes imprégnées de ces aprioris un accueil plutôt mitigé des juifs dans la Péninsule. Tiraillée entre ses intérêts économiques et culturels, qui l'amenaient à chercher à resserrer les liens avec ses "enfants perdus", pour reprendre la terminologie d'Ángel Pulido, fondateur du mouvement philosépharade, et ses réflexes de rejet des juifs, l'Espagne de Primo de Rivera promulgua donc un texte qu'elle choisit ensuite de n'assumer qu'en partie.

La France et l'Espagne, confrontée à des problématiques similaires quant à la situation juridique et administrative des communautés juives dans leurs protectorats respectifs dans les années 1920, ont été soumises à des campagnes similaires en faveur d'une naturalisation massive de ces groupes. Il est cependant remarquable que ces

38 A. A. Perl, «El advenimiento de la República en España y su relación con el judaísmo. II », Renacimiento de Israel, 199 (30 juin 1931) : 5.

${ }^{39}$ Ministerio de Estado, Estatuto militar sefardí (20 avril 1930), AMAE R. 1366/287. 
campagnes aient émané de groupes très différents : dans le cas français, c'est la presse communautaire, proche de l'Alliance Israélite Universelle, qui multiplie les articles défendant ce processus, en y mêlant un souci d'intégration de leurs coreligionnaires dans la communauté nationale, et l'intérêt patriotique. En Espagne, les tenants de cette nationalisation des juifs du protectorat appartiennent à une élite politico-intellectuelle, pour qui cette revendication s'intègre dans la définition d'un nationalisme reposant sur une forme d'impérialisme culturel, dans lequel s'intègre aussi, à la même époque, l'hispano-américanisme en plein essor. On notera cependant que, dans les deux cas, il s'agit d'une demande émanant de secteurs plutôt conservateurs sur un plan politique, et qui portent un regard plutôt condescendant sur les communautés juives du Maroc. Par ailleurs, tous choisissent comme modèle le Décret Crémieux de 1870, en oubliant les restrictions qui lui ont été apportées très rapidement, et le fait que dans les années 1920, il est encore loin d'être unanimement accepté dans les départements algériens.

Il faut enfin remarquer que le parallélisme avec l'Algérie n'était pas tout à fait pertinent, du fait du statut particulier de cette région. En effet, elle avait été de fait pleinement rattachée au territoire national en 1848, et divisée en trois départements. Les questions de naturalisation collective ou non des différents secteurs de la population ne concernaient finalement que l'administration française. Dans le cas du Protectorat marocain, il existait toujours une administration nationale marocaine, avec laquelle les deux puissances protectrices devaient composer, et qui régulièrement essaya de défendre son autorité, comme le prouvèrent les négociations répétées autour du statut de Tanger, par exemple. Il était donc difficile tant pour l'Espagne que pour la France de prendre des décisions unilatérales sur des questions relevant de compétences régaliennes, alors même que brûlaient les derniers feux de la révolte du Rif qui avait coûté si cher en vies humaines.

\section{BIBLIOGRAFÍA}

Abitbol, Michel, «De la tradition à la modernité: les juifs du Maroc», Judaïsmes: parcours dans la modernité. Diasporas, 27 (2016): 19-30. Doi: https://doi.org/10.4000/diasporas.439 
Aliberti, Davide, Sefarad Una comunidad imaginada (1924-2015), Madrid: Marcial Pons, 2018.

Allouche-Benayoun, Joëlle, «Les enjeux de la naturalisation des Juifs d'Algérie : du dhimmi au citoyen », en Le choc colonial et l'Islam, éd. par Pierre-Jean Luizard, 179-195. Paris : La Découverte, 2006.

Blévis, Laure, «La citoyenneté française au miroir de la colonisation: étude des demandes des "sujets français" en Algérie coloniale», Genèses, 53 (2003/4) : 25 47. https://www.cairn.info/revue-geneses-2003-4-page-25.htm

--. «L'invention de "l'indigène", Français non citoyen», en Histoire de l'Algérie à l'époque coloniale, éd. par Abderrahmane Bouchène et al., 212-218. Paris: La Découverte, 2014.

Cazes-Benatar, Hélène, «Sujets et protégés marocains», Le Monde juif, 39 (1951/1): 22. https://www.cairn.info/revue-le-monde-juif-1951-1-page-22.htm

González González, Irene, Escuela e ideología en el Protectorado español en el norte de Marruecos (1912-1956). Barcelona: Bellaterra-Casa Árabe, 2015

Kenbib, Mohamed, «La politique indigène du général Lyautey et les juifs marocains (1912-1925)», en De l'Atlas à l'Orient musulman, éd. par Dominique Avon et al., 63-84. Paris : Karthala, "Hommes et sociétés", 2011.

Laskier, Michael M., The Alliance Israélite Universelle and the Jewish Communities of Morocco 1862-1962, Albany: State University of New York Press, 1983.

Nataf, Claude, «La revendication de la nationalité française par les juifs de Tunisie (1881-1939)», en Nouvelle histoire des colonisations européennes (XIX $-X X^{e}$ siècles), éd. par Amaury Lorin et al., 117-128. Paris: Presses Universitaires de France, "Le Nœud gordien”, 2013.

Renucci, Florence, «Le débat sur le statut politique des israélites en Algérie et ses acteurs (1870-1943)», Contributions du séminaire sur les administrations coloniales (2009-2010), 31-49. Paris, 2010. https://halshs.archivesouvertes.fr/halshs-00599296

Touboul Tardieu, Eva, Séphardisme et Hispanité : l'Espagne à la recherche de son passé, Paris: PUPS, 2009.

Zafrani, Haïm, Deux mille ans de vie juive au Maroc: histoire et culture, religion et magie. Paris: Maisonneuve \& Larose, 1998. 